Thorax, 1980, 35, 959-960

\title{
Cardiac tamponade in acute pancreatitis
}

\author{
R WITHRINGTON AND P COLLINS
}

From the Stoke Mandeville Hospital, Aylesbury, Buckinghamshire

The complications of acute pancreatitis are uncommon, generally local, and carry a high mortality. ${ }^{1}$ We report a case of a remote complication, in which clinical deterioration resulted from cardiac tamponade.

\section{Case report}

A 37-year-old woman was admitted to hospital with severe epigastric pain. She gave a four-month history of intermittent epigastric discomfort aggravated by fats. This had proved unresponsive to cimetidine, carbenoxolone, and diazepam. She did not drink alcohol.

She was distressed, mildly jaundiced, and had marked abdominal tenderness. Her blood pressure was $130 / 70 \mathrm{mmHg}$ and her heart rate $80 / \mathrm{min}$. No abnormal cardiac signs were present on admission.

Her chest and plain abdominal radiographs were normal. The haemoglobin was $14 \cdot 3 \mathrm{~g} / 100 \mathrm{ml}$ and the white cell count $13100 / \mathrm{cu} \mathrm{mm}$. Biochemical investigations were as follows (normal results in brackets): serum amylase 4800 Somogyi units $/ 100 \mathrm{ml}(<160)$; serum alkaline phosphatase 13.5 KA units (4-13); serum bilirubin $60.2 \mathrm{~mol} / 1$ (5-17); SGPT $74 \mathrm{IU} / 1$ $(<40)$. Her serum calcium, blood glucose and blood urea were normal.

A diagnosis of acute pancreatitis was made. She was treated with analgesics and intravenous fluid. She developed pyrexia and paralytic ileus on the third day. A repeat chest radiograph revealed shadowing at the left base and she was treated with gentamicin and ampicillin. Chest pain and a pericardial friction rub developed on the fourth day. She became distressed and her systolic blood pressure fell to $80 \mathrm{mmHg}$ with pulsus paradoxus and a raised venous pressure. Chest radiograph now revealed an enlarged cardiac shadow and a left pleural effusion, but her ECG was normal.

Pericardial paracentesis was performed and $120 \mathrm{ml}$ of turbid amber fluid was removed. This produced rapid clinical improvement. The fluid contained a mixture of neutrophils and lymphocytes. Its protein concentration was $4.7 \mathrm{~g} / 100 \mathrm{ml}$. Culture was sterile and examination for malignant cells and LE cells was negative. Amylase was not measured.

Recovery was uneventful after pericardial paracentesis. A subsequent cholecystogram showed no concentration of dye by the gall bladder, but an intravenous cholangiogram revealed non-opaque gall stones in a contracted gall bladder.

Address for reprint requests: Dr P Collins, Stoke Mandeville Hospital, Aylesbury, Buckinghamshire.

\section{Discussion}

Pleural effusions, often rich in amylase, are not uncommon in acute pancreatitis. ${ }^{23}$ In contrast, pericardial effusion complicating acute pancreatitis is rare. Review of the literature has revealed three previous case reports, ${ }^{4-6}$ and one of these patients developed tamponade and needed resuscitation. ${ }^{5}$

The mechanism by which pericardial and pleural effusions accumulate in acute pancreatitis is unknown. Mitchells proposed chemical pericarditis and pleurisy as a result of the action of pancreatic enzymes transported from the pancreas by lymphatic vessels. Auger ${ }^{4}$ described necrosis of vascular walls in areas of fat necrosis in the subpericardial fat in his fatal case. Lipson and Stephenson ${ }^{6}$ considered chemical pericarditis to be improbable on the grounds that the pericardium in their patient appeared normal during open-chest resuscitation. However, they did not examine histological material to support their contention that the pericardium was normal. Also, they offered no evidence to support their hypothesis of fistulous connections between the abdominal and pericardial cavities as a source of the pericardial effusion.

Estimation of the amylase level in the pericardial fluid was made by Mitchell only. ${ }^{5}$ In his patient the amylase level in the pericardial effusion exceeded that in the serum by a factor of 22 . This favours some local mechanism as a source of the amylase, rather than the circulation, but does not distinguish between lymphatic transport and a fistula. Animal experiments, however, lend support to the theory of lymphatic transport. ${ }^{7}$

The limited histological evidence available is compatible with the theory that damage to the pericardial vessels by enzymes, or other products of pancreatic inflammation, may play a role in the development of the effusion. ${ }^{4} \mathrm{~A}$ common viral aetiology of the pancreatitis and pericarditis is unlikely, as adequate explanation for the development of pancreatitis was present in all cases-alcoholism, 56 carcinoma of the pancreas, ${ }^{4}$ and biliary tract disease in the present case.

The explanation for the frequency of pleural effusion in acute pancreatitis, compared with the apparent rarity of pericardial effusion, is difficult to understand, considering that the same mechanism has been postulated for their development, but may relate to the insensitivity of chest radiographs as a means of detecting small pericardial effusions. Prospective echocardiographic studies should provide a true estimate of the frequency of pericardial effusion. With the exception of the patient reported 
by Lipson and Stephenson, ${ }^{6}$ where the effusion was confined to the pericardium, all patients with pericardial effusions had simultaneous pleural effusions.

Although pericardial tamponade is rare it should be considered as an explanation of otherwise unexplained deterioration in a patient with acute pancreatitis.

\section{References}

1 Trapnell JE. Pancreatitis. $\mathrm{Br} J$ Hosp Med 1974; 12:193.

2 Hammarsten AF, Honska W, Limes BF

\section{Correspondence}

Mycoplasma pneumonia with fulminant evolution into diffuse interstitial fibrosis

Sir,-We have read with interest the report ${ }^{1}$ in which Mycoplasma pneumonia with fulminant evolution into diffuse interstitial fibrosis is considered to be the first well-documented case. In 1977, Mantz and coworkers ${ }^{2}$ published two well-documented cases of Mycoplasma pneumonia which developed into diffuse, interstitial pulmonary fibrosis within two to five weeks and, in February 1980, we reported the case of a healthy young woman who developed diffuse interstitial fibrosing pneumonia followed by refractory hypoxaemia. ${ }^{3}$ In this case, the diagnosis of Mycoplasma pneumonia was at first suspected from an elevated titre in the standard complement fixation test and then confirmed in the immunofluorescence study by the presence of granular deposits (Mycoplasma) in the macrophages of lung tissue. Our report appears to be the first one where the diagnosis has been confirmed with another method differing from the complement fixation test.

GUIDO DOMENIGHETTI, CLAUDE PERRET Department of Medicine, CHUV 1011 Lausanne, Switzerland

\section{References}

1 Kaufmann JM, Cuvelier CA, Van der Straeten M. Mycoplasma pneumonia with fulminant evolution into diffuse interstitial fibrosis. Thorax $1980 ; 35: 140-4$.

2 Mantz JM, Porte A, Stoekel ME, Batzenschlager A, Tempe JD, Jaeger A. Grippes malignes et pneumopathies à mycoplasma pneumoniae avec évolution fibrogène. Rev Fr Mal Respir 1977; 5:553-64.

3 Reigner Ph, Domenighetti G, Feihl F, Bonjour J$\mathrm{Ph}$, Perret $\mathrm{Cl}$. Syndrome de détresse respiratoire aigu sur infection à mycoplasme. Schweiz Med Wschr 1980; 110:220-3.
Pleural fluid amylase in pancreatitis and other disease. Am Rev Tuberc 1959; 79:606.

3 Goldman M, Goldman G, Fleischaer FG. Pleural fluid amylase in acute pancreatitis. $\mathbb{D}$ $N$ Engl J Med 1962; 266:715.

4 Auger C. Acinous cell carcinoma of pancreas $\tilde{\omega}^{\circ}$ with extensive fat necrosis. Arch Pathol 1947; 43:400.

5 Mitchell CE. Relapsing pancreatitis with $\vec{\overrightarrow{ }}$ recurrent pericardial and pleural effusions. $\vec{\omega}$ Ann Intern Med 1964; 60:1047.

6 Lipson JD, Stephenson HE. Pancreatitis com- $\vec{x}$ plicated by pericardial effusion and cardiac ${ }_{\omega}^{\times}$ tamponade. Arch Surg 1971; 103:414.

7 Perry TT. Role of lymphatic vessels in trans- $\vec{N}$ mission of lipase in disseminated pancreatic necrosis. Arch Pathol 1947; 43:456.

\section{Bone-scanning in hypertrophic osteoarthropathy}

SIR,-The recent report by CR Horn ${ }^{1}$ of a case of $\stackrel{\Phi}{7}$ symptomatic hypertrophic osteoarthropathy (HOA)음

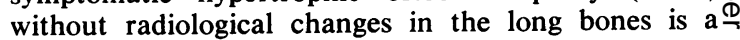
welcome reminder of this phenomenon.

I was surprised to see no mention of a radionucleotide bone-scan result as this investigation is a moresensitive indication of the presence of HOA than radiography. ${ }^{2}$ Occasionally the characteristic changes of HOA are found despite normal radiographic findings, ${ }^{2} 3$ These changes might well have been detected in this case. This investigation has also been shown $\mathbb{\Omega}$ to be of great value in differentiating the symptoms $\vec{F}$ of HOA from those of rheumatoid arthritis (particu- $\frac{0}{3}$ larly in those cases with joint swelling, morning stiff- $\frac{}{J}$ ness, elevated erythrocyte sedimentation rate, and a symptomatic response to anti-inflammatory drugs), ${ }^{4}{ }^{5}$ or osseous metastases (thereby influencing decisions on surgical intervention). ${ }^{3}{ }^{4}$ Involvement of "other" bones음 such as the mandible, maxillae, scapulae, patellae, ilii,, and ribs in this unusual disorder has also been de-o tected by this method. ${ }^{2}$

BWS ROBINSONO̊

Department of Respiratory Medicine Sir Charles Gairdner Hospitalo Queen Elizabeth II Medical Centre Nedlands 6009 응 Western Australia

\section{References}

1 Horn CR. Hypertrophic osteoarthropathy without N radiographic evidence of new bone formation.
Thorax 1980; 35:479.

2 Rosenthall L, Kirsh J. Observations on radiose nucleotide imagining in hypertrophic pulmonary osteoarthropathy. Radiol 1976; 120:359-62.

3 Stokes TC, Ell PJ, Deacon J. Diagnosis of hypertrophic pulmonary osteoarthropathy. $\mathrm{Br}$ Med $\mathrm{J}^{\circ}$ 1977; 2:1151-2.

4 Editorial. Finger clubbing and hypertrophic pulmonary osteoarthropathy. Br Med J 1977; 2:785.

5 Hammersten JF, O'Leary J. The features and significance of hypertrophic osteoarthropathye Arch Intern Med 1957; 99:431-41. 